\title{
SOME RESULTS AROUND QUADRATIC MAPS
}

\author{
Shiva SHEYBANI ${ }^{1}$, Mohsen Erfanian OMIDVAR ${ }^{2}$, Mahnaz KHANEHGIR ${ }^{3}$, \\ and Silvestru Sever DRAGOMIR ${ }^{4}$ \\ ${ }^{1,2,3}$ Department of Mathematics, Mashhad Branch, Islamic Azad University, Mashhad, IRAN \\ ${ }^{4}$ School of Computer Science and Mathematics Victoria University of Technology, PO Box \\ 14428, MCMC 8001, Victoria, AUSTALIA
}

\begin{abstract}
This paper dedicated to study quadratic maps. We present some new operator equalities and inequalities by using quadratic map in the framework of $\mathcal{B}(\mathscr{H})$. Applications for particular case of interest are also provided.
\end{abstract}

\section{Introduction AND PRELIMINARIES}

As customary, we reserve $\alpha$ for scalars and other capital letters denote general elements of the $C^{*}$-algebra $\mathcal{B}(\mathscr{H})$ of all bounded linear operator acting on Hilbert space $(\mathscr{H},\langle\cdot, \cdot\rangle)$. The absolute value of operator $A$ is denoted by $|A|=\left(A^{*} A\right)^{\frac{1}{2}}$, where $A^{*}$ stands for the adjoint of $A$. An operator $A$ is called positive (in symbol: $A \geq 0)$ if $\langle A x, x\rangle \geq 0$. A linear map $\phi: \mathcal{B}(\mathscr{H}) \rightarrow \mathcal{B}(\mathscr{H})$ is positive if $\phi(A) \geq 0$ whenever $A \geq 0$. More information on such maps can be found in [10, p. 18]. The study of linear maps on an algebra of bounded linear operators on a Hilbert space has been developed by many authors (see for instance [3, 5, 7, 13, 14]). Also, for a host of positive linear map inequalities, and for diverse applications of these inequalities, we refer to [8, 11, 15, and references therein. As is known to all, the linear property plays an important role to obtain this inequalities.

The motivation of this paper is to present some results concerning equalities and inequalities for maps without linear property on complex Hilbert spaces. In order to prove our main results, we need the following essential definitions. A map $\varphi: \mathcal{B}(\mathscr{H}) \times \mathcal{B}(\mathscr{H}) \rightarrow \mathcal{B}(\mathscr{H})$ is a sesquilinear, if satisfying the following conditions:

(1) $\varphi\left(\alpha A_{1}+\beta A_{2}, B\right)=\alpha \varphi\left(A_{1}, B\right)+\beta \varphi\left(A_{2}, B\right)$;

2020 Mathematics Subject Classification. Primary 42B10; Secondary 26A16.

Keywords and phrases. Quadratic map, operator inequalities, absolute value.

凶 shiva.sheybani95@gmail.com; math.erfanian@gmail.com (Corresponding author); khanehgir@mshdiau.ac.ir; sever.dragomir@vu.edu.au

(D) 0000-0002-7285-1571; 0000-0002-5395-8170; 0000-0002-7435-7307; 0000-0003-2902-6805.

Co 2020 Ankara University
Communications Faculty of Sciences University of Ankara-Series A1 Mathematics and Statistics 
(2) $\varphi\left(A, \alpha B_{1}+\beta B_{2}\right)=\bar{\alpha} \varphi\left(A, B_{1}\right)+\bar{\beta} \varphi\left(A, B_{2}\right)$;

for all $\alpha, \beta \in \mathbb{C}$ and $A_{1}, A_{2}, B_{1}, B_{2} \in \mathcal{B}(\mathscr{H})$. A sesquilinear form $\varphi$ is called positive if $\varphi(A, A) \geq 0$, for each $A \in \mathcal{B}(\mathscr{H})$. The sesquilinear form $\varphi$ is said to be symmetric if $\varphi(A, B)=\varphi(B, A)$ for all $A, B \in \mathcal{B}(\mathscr{H})$. The map $\Phi: \mathcal{B}(\mathscr{H}) \rightarrow$ $\mathcal{B}(\mathscr{H})$ defined by $\Phi(A)=\varphi(A, A)$, is called the quadratic map associated with $\varphi$. It can be easily verified that the definition of quadratic map is different from positive linear map. In fact, by using a sesquilinear map we create a quadratic map, that is not necessarily linear and positive.

The paper is organized in the following way: After this Introduction, in Section 2 we deduce some equalities. The parallelogram law is recovered (see Theorem 2.1 and 2.2 and some other interesting operator equalities are established. Afterward, in Section 3, we get an extension of some well known inequalities such as, triangle (Theorem 3.1) inequality. Especially, Bohr's inequality is generalized to the context of quadratic map (see Theorem 3.4). Some results concerning this inequality are surveyed (see Corollary 3.5 and 3.6). In Section 4 before closing the paper, we give an application of our results in the previous sections. We show that our results are a generalization of some well known works due to Fujii [9] and Hirzallah [12.

\section{Some EqUalities For QUADRATiC MAPS}

Here and throughout, $\Phi$ stands for the quadratic map. Our first main result in this section reads as follows.

Theorem 2.1. Let $A, B \in \mathcal{B}(\mathscr{H})$. Then

$$
\Phi(A+B)+\Phi(A-B)=2(\Phi(A)+\Phi(B)) .
$$

Proof. We observe that

$$
\varphi(A+B, A+B)=\varphi(A, A)+\varphi(A, B)+\varphi(B, A)+\varphi(B, B) .
$$

Replace $B$ by $-B$ in the above equality, we deduce

$$
\varphi(A-B, A-B)=\varphi(A, A)-\varphi(A, B)-\varphi(B, A)+\varphi(B, B) .
$$

By adding 2.2 and $(2.3)$, we obtain desired result (2.1).

The following generalization of the parallelogram law holds.

Theorem 2.2. Let $A, B \in \mathcal{B}(\mathscr{H})$ and $0 \neq t \in \mathbb{R}$. Then

$$
\Phi(A+B)+\frac{1}{t} \Phi(t A-B)=(1+t) \Phi(A)+\left(1+\frac{1}{t}\right) \Phi(B) .
$$


Proof. We observe that

$$
\begin{aligned}
\Phi(A & +B)+\frac{1}{t} \Phi(t A-B) \\
= & \Phi(A)+\Phi(B)+\varphi(A, B)+\varphi(B, A) \\
& +t \Phi(A)+\frac{1}{t} \Phi(B)-\varphi(A, B)-\varphi(B, A) \\
= & (1+t) \Phi(A)+\left(1+\frac{1}{t}\right) \Phi(B) .
\end{aligned}
$$

which proves the theorem.

Remark 2.3. Assume that $\varphi$ is a positive sesquilinear form. If $0<t \leq 1$, then $\frac{1}{t} \geq 1$, so that the second term $\frac{1}{t} \Phi(t A-B)$ of the left side of the equality $[2.4$ is greater that $\Phi(t A-B)$. Hence we have

$$
\Phi(A \mp B)+\Phi(t A \pm B) \leq(1+t) \Phi(A)+\left(1+\frac{1}{t}\right) \Phi(B) .
$$

Similarly, if either $t \geq 1$ or $t<0$, then

$$
\Phi(A \mp B)+\Phi(t A \pm B) \geq(1+t) \Phi(A)+\left(1+\frac{1}{t}\right) \Phi(B) .
$$

The following result can be regarded as an extension of the well-known Apollonius's identity (see, e.g., 2, Lemma 2.12]).

Theorem 2.4. Let $A, B, C \in \mathcal{B}(\mathscr{H})$. Then

$$
\Phi(A-B)=2 \Phi(C-A)+2 \Phi(C-B)-4 \Phi\left(C-\frac{A+B}{2}\right) .
$$

Proof. By Theorem 2.1, we have

$$
\begin{aligned}
\Phi\left(C-\frac{A+B}{2}\right) & =\Phi\left(\frac{C}{2}-\frac{A}{2}+\frac{C}{2}-\frac{B}{2}\right) \\
& =2\left[\Phi\left(\frac{C}{2}-\frac{A}{2}\right)+\Phi\left(\frac{C}{2}-\frac{B}{2}\right)\right]-\Phi\left(\frac{B}{2}-\frac{A}{2}\right) \\
& =\frac{1}{2}[\Phi(C-A)+\Phi(C-B)]-\frac{1}{4} \Phi(B-A) .
\end{aligned}
$$

which is clearly equivalent to 2.5 .

The following result concerning the quadratic maps may be stated.

Theorem 2.5. Let $A, B \in \mathcal{B}(\mathscr{H})$. Let $\varphi$ be symmetric sesquilinear form and $\Phi(A)=\Phi(B)$. Then for each $\pm 1,0 \neq \alpha \in \mathbb{R}$ we have

$$
\Phi(A+\alpha B)=\Phi(B+\alpha A) .
$$


Proof. One can easily see that

$$
\begin{aligned}
\Phi(A+\alpha B) & =\Phi(A)+2 \alpha \varphi(A, B)+\alpha^{2} \Phi(B) \\
& =\Phi(B)+2 \alpha \varphi(B, A)+\alpha^{2} \Phi(A) \\
& =\Phi(B+\alpha A) .
\end{aligned}
$$

Therefore we obtain the desired equality (2.6).

Theorem 2.6. Let $A, B, C \in \mathcal{B}(\mathscr{H})$ such that $A+B+C=0$, and let $\varphi$ be a symmetric sesquilinear form and $\Phi(A)=\Phi(B)$. Then

$$
\Phi(A-C)=\Phi(B-C) .
$$

Proof. By easy computation we have

$$
\begin{aligned}
& \Phi(A-C)+\Phi(A-B) \\
& \quad=2 \Phi(A)+\Phi(C)+\Phi(B)-2 \varphi(A, B+C) \\
& \quad=4 \Phi(A)+\Phi(C)+\Phi(B) .
\end{aligned}
$$

Also

$$
\Phi(B-C)+\Phi(A-B)=4 \Phi(B)+\Phi(C)+\Phi(A) .
$$

Hence

$$
\Phi(A-C)=\Phi(B-C) .
$$

The results in the following proposition is derived from the Theorem 2.6.

Proposition 2.7. Let $A, B, C, D \in \mathcal{B}(\mathscr{H})$ such that $A+B+C+D=0$, and let $\varphi$ be a symmetric sesquilinear form and $\Phi(A)=\Phi(B), \Phi(C)=\Phi(D)$. Then

$$
\Phi(A-C)=\Phi(B-D),
$$

and

$$
\Phi(B-C)=\Phi(A-D) .
$$

Proof. It is easy to obtain that

$$
\begin{aligned}
& \Phi(A-C)+\Phi(A-B)=2 \Phi(A)+\Phi(C)+\Phi(B)-2 \varphi(A, C+B), \\
& \Phi(B-D)+\Phi(A-B)=2 \Phi(B)+\Phi(C)+\Phi(A)-2 \varphi(B, A+D) .
\end{aligned}
$$

Subtracting and using the hypothesis, this gives

$$
\begin{aligned}
\Phi(A-C)-\Phi(B-D) & =2 \varphi(B, A+D)-2 \varphi(A, C+B) \\
& =2 \varphi(B, A+D)+2 \varphi(A, A+D) \\
& =2 \varphi(A+B, A+D) .
\end{aligned}
$$

Now

$$
\varphi(A+B, A)=\Phi(A)+\varphi(B, A)=\Phi(B)+\varphi(A, B)=\varphi(A+B, B),
$$


and

$$
\varphi(A+B, D)=-\varphi(C+D, D)=-\varphi(C+D, C)=\varphi(A+B, C) .
$$

Therefore

$$
\varphi(A+B, A+D)=\varphi(A+B, B+C)=-\varphi(A+B, A+D)=0,
$$

which implies that

$$
\Phi(A-C)=\Phi(B-D) .
$$

We can easily also check that

$$
\Phi(B-C)=\Phi(A-D) .
$$

\section{Some inequalities For QUAdratiC MAPS}

The following simple result is of interest in itself as well:

Theorem 3.1. Let $A, B \in \mathcal{B}(\mathscr{H})$ and $\Phi$ be a positive quadratic map associated with $\varphi$ such that $\varphi$ is symmetric. Then

$$
4 \operatorname{Re} \varphi(A, B) \leq \Phi(A+B) \leq 2(\Phi(A)+\Phi(B)) .
$$

Proof. Since $\Phi(A-B) \geq 0$, then

$$
\Phi(A)+2 \operatorname{Re} \varphi(A, B)+\Phi(B) \geq 4 \operatorname{Re} \varphi(A, B),
$$

therefore

On the other hand

$$
\Phi(A+B) \geq 4 \operatorname{Re} \varphi(A, B)
$$

$$
\Phi(A)+\Phi(B) \geq 2 \operatorname{Re} \varphi(A, B)
$$

then

$$
2(\Phi(A)+\Phi(B)) \geq \Phi(A)+\Phi(B)+2 \operatorname{Re} \varphi(A, B),
$$

So

$$
2(\Phi(A)+\Phi(B)) \geq \Phi(A+B) .
$$

By (3.2) and (3.3) we deduce the desired result 3.1).

It is worth to mention that the right side of inequality 3.1 is an extension of the triangle inequality.

Corollary 3.2. Let $A, B, C \in \mathcal{B}(\mathscr{H})$ and $\Phi$ be a positive quadratic map associated with $\varphi$ such that $\varphi$ is symmetric. Then

$$
\Phi(A-C) \leq 2(\Phi(A-B)+\Phi(B-C)) .
$$

The forthcoming theorem gives an upper bound for $\Phi(A+B)$.

Theorem 3.3. Let $A, B \in \mathcal{B}(\mathscr{H})$ and $\Phi$ be a positive quadratic map associated with $\varphi$ such that $\varphi$ is symmetric and $\Phi(A)=\Phi(B)$. Then for each $0 \neq \alpha \in \mathbb{R}$,

$$
\Phi(A+B) \leq \Phi\left(\alpha A+\alpha^{-1} B\right)
$$


Proof. We know that for any real numbers $\alpha \neq 0,\left(\alpha-\alpha^{-1}\right)^{2} \geq 0$ so $\alpha^{2}+\alpha^{-2} \geq 2$. Using the fact that $\Phi(A)=\Phi(B)$, one has

$$
\begin{aligned}
\Phi\left(\alpha A+\alpha^{-1} B\right) & =\alpha^{2} \Phi(A)+2 \varphi(A, B)+\alpha^{-2} \Phi(B) \\
& =\left(\alpha^{2}+\alpha^{-2}\right)\left(\frac{\Phi(A)+\Phi(B)}{2}\right)+2 \varphi(A, B) \\
& \geq \Phi(A)+\Phi(B)+2 \varphi(A, B) \\
& =\Phi(A+B) .
\end{aligned}
$$

This completes the proof of Theorem 3.3 .

Several authors discussed operator version of Bohr inequality (see for instance [4). In the following, we give a unified version of Bohr inequality.

Theorem 3.4. Let $A, B \in \mathcal{B}(\mathscr{H})$ and $p, q>1$ with $\frac{1}{p}+\frac{1}{q}=1, p \leq q$, and let $\Phi$ be a positive quadratic map. Then

$$
\Phi(A-B)+\Phi((1-p) A-B) \leq p \Phi(A)+q \Phi(B) .
$$

Proof. By easy computation observe that

$$
\begin{aligned}
& p \Phi(A)+q \Phi(B)-\Phi(A-B)-\Phi((1-p) A-B) \\
& =(2-p)(p-1) \Phi(A)+(q-2) \Phi(B)-(p-2)(\varphi(A, B)+\varphi(B, A)) \\
& =(2-p)(p-1) \Phi(A)+\left(\frac{2-p}{p-1}\right) \Phi(B)+(2-p)(\varphi(A, B)+\varphi(B, A)) \\
& =(2-p) \Phi\left(\sqrt{p-1} A+\frac{1}{\sqrt{p-1}} B\right) \\
& \geq 0
\end{aligned}
$$

where the last inequality follows from the fact that $p \leq q$ and so the proof is complete.

The following corollary is a natural consequence of the above result.

Corollary 3.5. Let $A, B \in \mathcal{B}(\mathscr{H})$ and $p, q>1, \frac{1}{p}+\frac{1}{q}=1$, and let $\Phi$ be a positive quadratic map. Then

$$
\Phi(A-B) \leq p \Phi(A)+q \Phi(B) .
$$

The next results follows by applying Corollary 3.5. first to the operators $A, B$ and second to the operators $A,-B$.

Corollary 3.6. Let $A, B \in \mathcal{B}(\mathscr{H})$ and $\Phi$ be a positive quadratic map. Then for any $p>1$,

$$
\pm(\varphi(A, B)+\varphi(B, A)) \leq(p-1) \Phi(A)+\frac{1}{p-1} \Phi(B) .
$$




\section{SPECial CASE}

For two bounded linear operators $A, B \in \mathcal{B}(\mathscr{H})$, we define the map $\varphi: \mathcal{B}(\mathscr{H}) \times$ $\mathcal{B}(\mathscr{H}) \rightarrow \mathcal{B}(\mathscr{H})$, with $\varphi(A, B)=B^{*} A$. This leads to $\varphi(A, A)=|A|^{2}$. It is obvious that $\varphi(A, B)$, is linear in the first variable and conjugate-linear in the second. For this we first observe from (2.1) the classic parallelogram law for operators.

$$
|A+B|^{2}+|A-B|^{2}=2\left(|A|^{2}+|B|^{2}\right) .
$$

We have from 2.5, the following well known equality

$$
|A-B|^{2}=2|C-A|^{2}+2|C-B|^{2}-4\left|C-\frac{A+B}{2}\right|^{2} .
$$

The following generalization of parallelogram law is derived from inequality (2.4), which is obtained in [9, Theorem 4.1].

$$
|A+B|^{2}+\frac{1}{t}|t A-B|^{2}=(1-t)|A|^{2}+\left(1+\frac{1}{t}\right)|B|^{2} .
$$

We also remark that, Corollary[2.3 is equivalent to [9, Theorem 3.1] by interchanging $\varphi(A, B)=B^{*} A$.

Also, Theorem 3.4 becomes

$$
|A-B|^{2}+|(1-p) A-B|^{2} \leq p|A|^{2}+q|B|^{2} .
$$

This result was obtained in [12, Theorem 1].

Acknowledgment. The authors would like to express their hearty thanks to the referee for their valuable comments.

\section{REFERENCES}

[1] Amir, D., Characterizations of inner product spaces, Birkhauser Verlag, 1986.

[2] Bauschke, H.H., Combettes, P.L., Convex Analysis and Monotone Operator Theory in Hilbert Spaces, Springer, New York, 2011.

[3] Bhatia, R., Sharma, R., Some inequalities for positive linear maps, Linear Algebra Appl., 436(6) (2012), 1562-1571.

[4] Cheung, W.S., Pečarić, J., Bohr's inequalities for Hilbert space operators, J. Math. Anal. Appl., 323(1) (2006), 403-412.

[5] Choi, M.D., Hadwin, D., Nordgren, E., Radjavi, H., Rosenthal, P., On positive linear maps preserving invertibility, J. Funct. Anal. 59(3) (1984), 462-469.

[6] Cvetkovski, Z., Inequalities: Theorems, Techniques and Selected Problems, Springer Science \& Business Media, 2012.

[7] Evans, D.E., Positive linear maps on operator algebras, Comm. Math. Phys., 48(1) (1976), $15-22$.

[8] Fu, X., Some generalizations of operator inequalities, J. Math. Inequal., 9(1) (2015), 101-105.

[9] Fujii, M., Zuo, H., Matrix order in Bohr inequality for operators, Banach J. Math. Anal., 1 (2010), 21-27.

[10] Furuta, T., Mićić Hot, J., Pečarić, J., Seo, Y., Mond-Pečarić method in operator inequalities, Monographs in Inequalities, Zagreb, 2005. 
[11] Gumus I.H., A note on a conjecture about Wielandt's inequality, Linear Multilinear Algebra, 63(9) (2015), 1909-1913.

[12] Hirzallah, O., Non-commutative operator Bohr inequality, J. Math. Anal. Appl., 282(2) (2003), 578-583.

[13] Lin, M., On an operator Kantorovich inequality for positive linear maps, J. Math. Anal. Appl., 402(1) (2013), 127-132.

[14] Størmer, E., Positive linear maps of operator algebras, Acta Math., 110(1) (1963), 233-278.

[15] Zhang, P., More operator inequalities for positive linear maps, Banach J. Math. Anal., 9(1) (2015), 166-172. 